\title{
Hydroxyapatite nanoparticles modified by branched polyethylenimine are effective non-viral vectors for siRNA transfection of hepatoma cells in vitro
}

\author{
XIAO-LIN XU ${ }^{1 *}$, HAI-YUN YANG ${ }^{1 *}$, BING OU $^{1 *}$, SHU-DONG LIN ${ }^{2}$, HUAN WU $^{1}$, \\ WANG HE $^{3}$, QIONG-CHAO JIANG ${ }^{1}$, BAO-MING LUO ${ }^{1}$ and GAO-PENG LI ${ }^{1}$ \\ ${ }^{1}$ Department of Ultrasound, Sun Yat-Sen Memorial Hospital, Sun Yat-Sen University; \\ ${ }^{2}$ Guangzhou Institute of Chemistry, Chinese Academy of Sciences; ${ }^{3}$ Department of Urology, \\ Sun Yat-Sen Memorial Hospital, Sun Yat-Sen University, Guangzhou, Guangdong, P.R. China
}

Received November 26, 2014; Accepted January 21, 2015

DOI: 10.3892/ijo.2015.2918

\begin{abstract}
Small interfering RNA (siRNA) technology is a powerful tool in biomedical research and holds great potential for RNA interference-based therapies for HIV, hepatitis and cancer. However, the absence of a safe and efficient method for the delivery of siRNA has become a bottleneck for their development. Nanocrystallized hydroxyapatite (nHAP) appears to be an optimal candidate non-viral gene vector for several reasons, including its good biocompatibility and ease of production, however, nHAP microemulsions cannot remain monodispersed for long periods of time. Due to their high surface energy, nHAP particles gradually aggregate into large ones that are difficult for the cell to take up. To overcome this we modified nHAP with polyethylenimine (PEI) to generate a compound (MnHAP) with a tight size-distribution of $<200 \mathrm{~nm}$. The positive surface potential of MnHAP inhibited particle aggregation and thus made it easier to conjugate more siRNA. The transfection efficiency of MnHAP/fluorescent FAM-labeled siRNA complex was tested using flow cytometry, and the transfected cells were observed using fluorescence microscopy. The cytotoxicity of MnHAP/siRNA complexes to the human liver cancer cell line BEL-7402 was assessed in vitro by a formazan dye assay. Our results show that the in vitro transfection efficiency of MnHAP/siRNA was equivalent to that of the commercially available transfection
\end{abstract}

Correspondence to: Dr Bao-Ming Luo or Dr Gao-Peng Li, Department of Ultrasound, Sun Yat-Sen Memorial Hospital of Sun Yat-Sen University, 107 Yanjiangxi Road, Yuexiu District, Guangzhou, Guangdong 510120, P.R. China

E-mail: bmluo2005@126.com

E-mail: ligaopeng@medmail.com.cn

*Contributed equally

Key words: nanoparticles, durapatite, neoplasms, therapy, transfection agent Lipofectamine ${ }^{\circledR} 2000$, but with decreased cytotoxicity. The MnHAP nanoparticles were also able to deliver siRNA for silencing of glyceraldehyde-3-phosphate dehydrogenase (GAPDH) in BEL-7402 cells, which supports that MnHAP might be a promising non-viral vector for biomedical research and gene delivery.

\section{Introduction}

In the past decade, RNA interference (RNAi), a posttranscriptional gene silencing technology, has been used widely in biomedical research $(1,2)$. However, the progress of in vivo studies using small interfering RNA (siRNA) has been slow because of the lack of safe and efficient gene delivery vehicles. Viral vectors are efficient for gene transfection, although they present risks, including inflammation and immunogenicity (3). Non-viral vectors, in contrast, are less toxic and immunogenic, but their transfection efficiency and the resulting gene expression changes are lower (4). In recent years, nanomedicine carboxy incorporating the advantages of nanotechnology in medicine, has attracted great deal of research interest. Hydroxyapatite (HAP) with the molecular formula $\mathrm{Ca}_{10}\left(\mathrm{PO}_{4}\right)_{6}(\mathrm{OH})_{2}$, is the main inorganic component of bones and teeth (durapatite), and has been used as a repair material for a long time in medicine $(5,6)$. HAP nanoparticles (nHAP) have, owing to their good biocompatibility (7), found utility in many applications, such as diagnostic probes (8), cancer treatments (9), delivery vectors for drugs, genes and other agents (10-14). Unfortunately, the use of nHAP for gene delivery is still limited due to particle aggregation, which results from the high interfacial free energy (13).

Aggregation of nHAP results in poorer cellular uptake (4) and the larger size of nanoparticles may trigger immunogenic responses in the body (15). This problem also impairs the colloid stability of nHAP, such that good transfection efficiency is possible for a short time only (16). Despite numerous fabrication processes being tested (17-19), it is still difficult to produce homogeneous and monodispersed nHAP. The positive charge of nHAP can improve the viability and proliferation of transfected cells, and can also make the colloid stable $(20,21)$. 
Some scientists have modified the surface charge of nHAP with $\mathrm{Ca}^{2+}, \mathrm{Mg}^{2+}$ and poly-L-lysine (PLL) $(10,22)$, however, the results indicated that the transfection efficiency remained low. PEI has been widely used to modify non-viral vectors for gene delivery and has led to high transfection efficiencies (23-27). Inspired by these findings, we modified nHAP using branched PEI in this study to increase the strength of heighten its surface charge and therefore improve its stability and the transfection efficiency. Our compound was also evaluated in vitro for its transfection efficiency and cytotoxicity.

\section{Material and methods}

Preparation of PEI modified $n H A P(M n H A P)$. HAP nanoparticles (nHAP) were purchased from Nanjing Emperor Nano Material Co. Ltd. (Nanjing, China). To overcome the disadvantages of nHAP as a gene delivery vector, we modified nHAP using branched PEI to prepare a new nanoparticle (which we named MnHAP), according to the protocol described by Ashokan et al (28) with some modifications. In brief, $200 \mathrm{mg}$ nHAP powder was dissolved in double distilled water and stirred at $1200 \mathrm{rpm} / \mathrm{min}$ for $12 \mathrm{~h}$. Then, PEI (MW $25 \mathrm{kDa}$, Sigma-Aldrich, St. Louis, MO, USA) was added to the nHAP microemulsion and stirring was continued for another $12 \mathrm{~h}$, all at room temperature. The generated MnHAP microemulsion was centrifuged at $5000 \mathrm{rpm}$ for $30 \mathrm{~min}$ after ultrasonification at $20 \mathrm{KHz}$ for $5 \mathrm{~min}$. The supernatant was collected for $48 \mathrm{~h}$ dialysis to remove the unreacted PEI. The resulting sample was lyophilized and stored at room temperature.

Characterization of MnHAP. The particle size distribution and zeta potential of MnHAP preparations were measured by Malvern Zetasizer Nano ZS90 (Malvern Instruments, Ltd., Malvern, UK). The morphology of the nanoparticles was examined using a Hitachi H-7650 transmission electron microscope at $80 \mathrm{kV}$.

Gel retardation assay. Different amounts of $\operatorname{MnHAP}(\mu \mathrm{g})$ were mixed with a fixed amount $(0.25 \mu \mathrm{g})$ of negative control siRNA (sense: 5'-UUC UCC GAA CGU GUC ACG UTT-3', antisense: 5'-ACG UGA CAC GUU CGG AGA ATT-3', Shanghai GenePharm Co., Ltd) according to the MnHAP:siRNA weight ratios $(0: 1,0.2: 1,0.4: 1,1: 1,2: 1,4: 1)$ by gentle pipetting for $30 \mathrm{~min}$ at room temperature. The above six mixtures were then electrophoresed on a $3 \%$ agarose gel at $100 \mathrm{~V}$ for $20 \mathrm{~min}$. The bands were visualized under a UV transilluminator.

Cell culture. The human liver cancer cell line BEL-7402 was obtained from the Laboratory Animal Center of Sun Yat-sen University of China. The cells were grown in RPMI-1640 (Gibco, USA) supplemented with $10 \%$ fetal bovine serum (FBS, Gibco) under $5 \% \mathrm{CO}_{2}$ in air at $37^{\circ} \mathrm{C}$.

Transfection efficiency of MnHAP conjugated with FAM-labeled siRNA. For transfection efficiency analysis, BEL-7402 cells were plated in 6-well plates at a density of $5 \times 10^{5} /$ well and allowed to adhere for $24 \mathrm{~h}$. Green fluorescent negative-control siRNA (FAM-siRNA) $(2.5 \mu \mathrm{g})$ and $5.0 \mu \mathrm{g}$ MnHAP, or $5 \mu 1$ Lipofectamine 2000 were used per well (sense: 5'-UUC UCC GAA CGU GUC ACG UTT-3'; antisense: 5'-ACG
UGA CAC GUU CGG AGA ATT-3', Shanghai GenePharm Co., Ltd). At $4 \mathrm{~h}$ after transfection with FAM-siRNA, MnHAP/FAM-siRNA complexes and Lipofectamine 2000/ FAM-siRNA complexes, cells were harvested and analyzed by flow cytometry to determine the percentage of FAM-positive cells. A Nikon Eclipse TE300 fluorescent microscope (Nikon, Tokyo, Japan) was used for cell observations and recording images after washing cells 3 times with PBS to reduce background fluorescence.

Cytotoxicity evaluation. A formazan-based assay (MTT assay, Sigma-Aldrich) was employed to determine the cytotoxicity of the MnHAP/siRNA mixture (weight ratio at 2:1). BEL-7402 cells were seeded in 96-well plates at a density of $10^{4} /$ well with $100 \mu \mathrm{l}$ culture medium. Cells were incubated for $24 \mathrm{~h}$ to allow complete adherence then the medium was replaced with fresh medium containing siRNA alone, MnHAP/siRNA complexes and Lipofectamine/siRNA complexes respectively; control wells received fresh medium. For each well, the amount of siRNA was $0.25 \mu \mathrm{g}$ and the amount of MnHAP was $0.25 \mu \mathrm{g}$ or $0.5 \mu 1$ Lipofectamine. Four replicates of each condition were present and analyzed simultaneously.

To assess how treatments affected the cell number, medium in each well was aspirated after $4 \mathrm{~h}$ incubation and $100 \mu \mathrm{l}$ fresh medium was added, followed by a further $24 \mathrm{~h}$ incubation after which time $10 \mu \mathrm{l}$ MTT $(5 \mathrm{mg} / \mathrm{ml}$, MP Biomedicals, USA) was added to each well and the cells were again incubated in a $\mathrm{CO}_{2}$ incubator for $4 \mathrm{~h}$. The supernatant containing MTT substrate was aspirated and $100 \mu \mathrm{l}$ DMSO added to dissolve the formazan deposits. The absorbance at $495 \mathrm{~nm}$ was measured on a spectrophotometer (Spectra Max M5, Molecular Devices), and the relative cell viability (\%) was calculated as the percentage of absorbance of the treated cells sample relative to that of the untreated control cells.

Western blot analysis. BEL-7402 cells were harvested $48 \mathrm{~h}$ after siRNA transfection and lysed with lysis buffer for $30 \mathrm{~min}$ on ice. Total protein concentration was determined using a BCA protein assay. For western blotting, protein extracts were electrophoresed on SDS-PAGE gels and transferred to nitrocellulose membranes. After blocking with 5\% non-fat milk powder, the membrane was first incubated overnight at $4^{\circ} \mathrm{C}$ with a mouse monoclonal antibody against GAPDH (Abcam). The blots were then washed and incubated with a goat anti-mouse IgG secondary antibody (Abcam). Bands were visualized using an ECL detection kit (Millipore) and intensities analyzed using Image $\mathbf{J}$ software. The relative expression of GAPDH was determined by dividing the densitometric value of the GAPDH band by that for its control (Tubulin).

Statistical analyses. All in vitro assays were performed at least twice independently, with 3 or 4 replicates of each data point. Student's t-test was employed for statistical analysis using the SPSS 13.0 software package (SPSS Inc., Chicago, IL, USA).

\section{Results and Discussion}

Preparation and characterization of $M n H A P$. To prevent nHAP aggregation and enhance transfection efficiency, nHAP was coated with branched PEI using published reaction conditions 
A

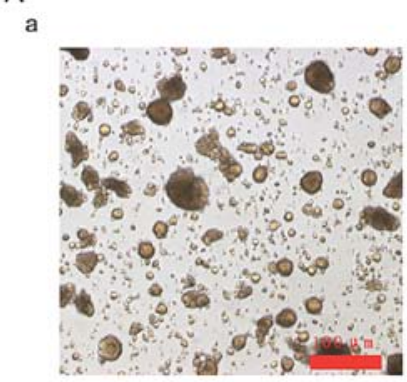

b

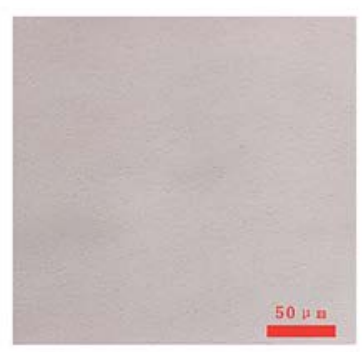

B

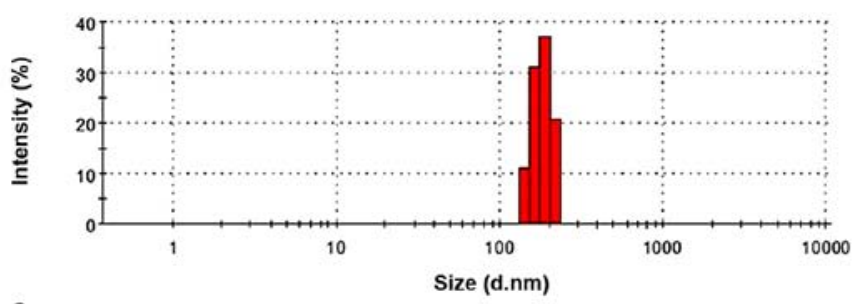

C

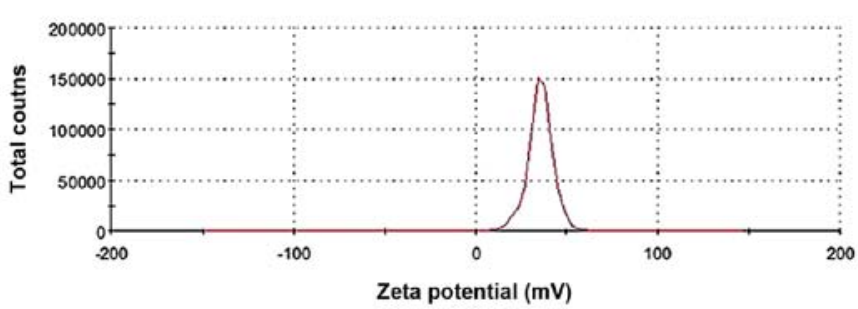

D

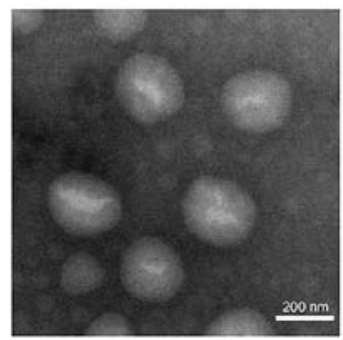

Figure 1. Characterization of MnHAP. (A) (a) Light microscopy (x200 magnification) showed that nHAP particles were of different sizes, and aggregated immediately in form of solution. (b) Light microscopy (x400 magnification) $24 \mathrm{~h}$ after the MnHAP microemulsion was prepared, showed that MnHAP particles remained evenly dispersed. (B) The size distribution of MnHAP particles was $<200 \mathrm{~nm}$, and the mean diameter was $184.5 \mathrm{~nm}$. (C) Zeta potential measurement of MnHAP in double distilled water. The surface of MnHAP showed a high positive charge of $+35.4 \mathrm{mV}$. (D) Transmission electron microscopy (TEM) at x1,5000 magnification demonstrated that MnHAP particles were of uniform size, spherical morphology and with smooth surfaces in accordance with the data in (A and B).

to generate MnHAP (28). The appearance of the newly formed MnHAP microemulsion was clear/milky and appearance was stable for more than 7 days without formation of precipitates, whereas nHAP alone sank quickly from the solution and formed aggregates. As seen using light microscopy, the particle dispersion of MnHAP was better than that of nHAP (Fig. 1A) and their average size was $184.5 \pm 20.5 \mathrm{~nm}$ (Fig. 1B). Surface charge is an important property of nanoparticles. When it exceeds $\pm 30 \mathrm{mV}$, particles in suspension could be kept well-dispersed and stably suspended (21). The surface charge of nHAP without modification is $-10.47 \mathrm{mV}$ (data not shown),

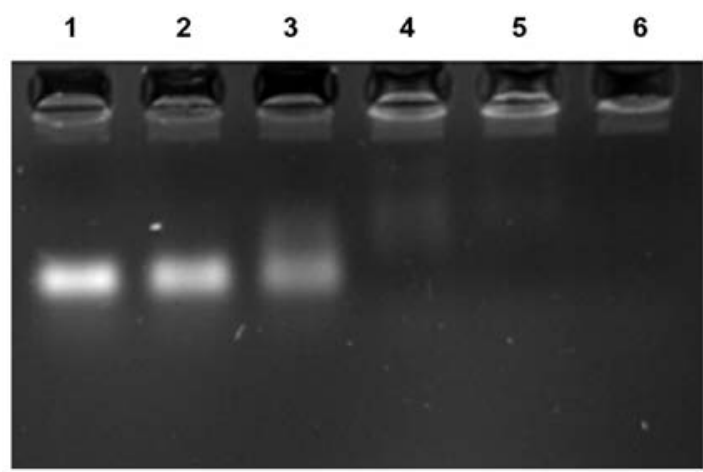

Figure 2. Gel retardation assay of MnHAP/siRNA complexes. Lane 1, free negative control siRNA $(0.25 \mu \mathrm{g})$; lane 2, MnHAP/siRNA complex with a weight ratio of $0.2: 1$; lane 3, MnHAP/siRNA at 0.4:1; lane 4, MnHAP/siRNA at $1: 1$; lane $5, \mathrm{MnHAP} / \mathrm{siRNA}$ at $2: 1$; lane $6, \mathrm{MnHAP} /$ siRNA at $4: 1$.

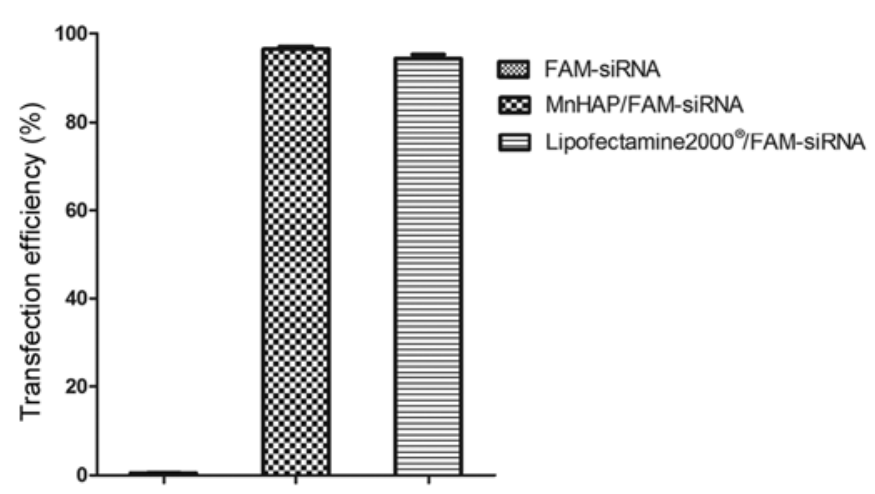

Figure 3. Comparison of gene transfection efficiency of various FAM-siRNA formulations after $4 \mathrm{~h}$ by flow cytometry. The amount of FAM-siRNA used in each group was $2.5 \mu \mathrm{g}$ and the ratio of MnHAP to FAM-siRNA was fixed at $2: 1$.

which implies that it is difficult for the dispersed particles to remain stable. In the present study, the measurement of surface zeta potential of MnHAP revealed a positive surface charge of $+35.4 \mathrm{mV}$, which not only enables the particles to avoid agglomeration but also facilitates binding to the negatively charged siRNA to form MnHAP/siRNA complexes (Fig. 1C). Moreover, when imaged at x1,5000 magnification using transmission electron microscopy (TEM) MnHAP particles were of uniform size and spherical with smooth surfaces (Fig. 1D).

Gel retardation assay. To assess the siRNA binding efficiency of MnHAP, a gel retardation assay was carried out at six weight ratios of MnHAP to siRNA while maintaining the amount of siRNA. Fig. 2 illustrates that with the increase of MnHAP/ siRNA weight ratio, the migration of siRNA bands was significantly retarded. When the ratio of the MnHAP/siRNA was $\geq 2: 1$, no free siRNA could be detected, which indicated that the siRNA was completely confined to the sample well. Therefore, the 2:1 ratio could be the minimal ratio for siRNA being totally bound by MnHAP and this ratio was used in the subsequent experiments.

Transfection efficiency determined by FAM-labeled control siRNA. The in vitro transfection studies on BEL-7402 cells 
A

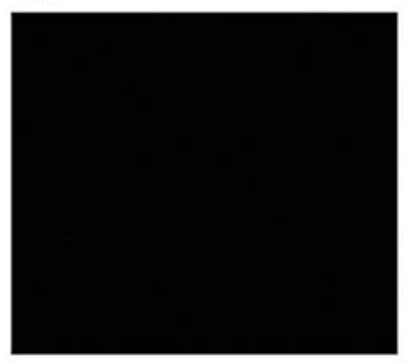

B

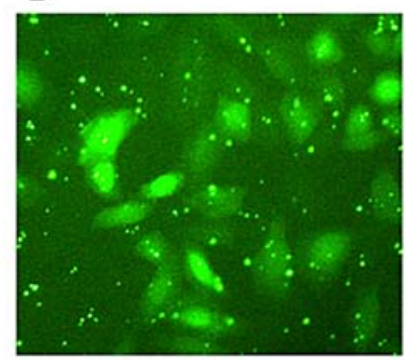

C

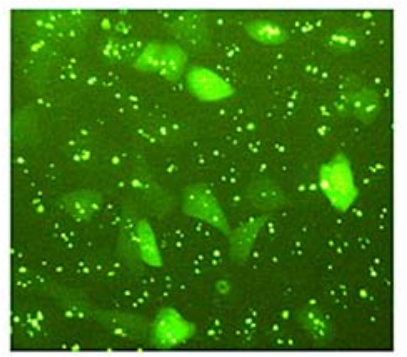

Figure 4. Observation of FAM-positive cells with fluorescence microscope at x200 magnification. The FAM-positive cells were directly visualized and the transfected cells with green granules in cell cytoplasm and intact cellular structures were counted in the same field. (A) FAM-siRNA, (B) MnHAP/FAMsiRNA, (C) Lipofectamine ${ }^{\circledR}$ 2000/FAM-siRNA.

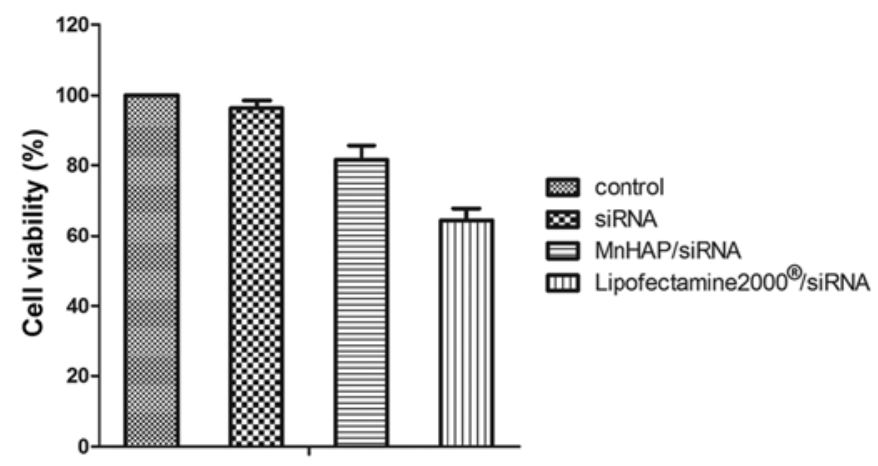

Figure 5. Cell viability analysis. BEL-7402 cells were treated with siRNA alone, MnHAP/siRNA complexes or Lipofectamine ${ }^{\circledR} 2000 /$ siRNA complexes for $4 \mathrm{~h}$ and incubated for $24 \mathrm{~h}$. The experiments were done with four replicates and the mean and standard error are shown.

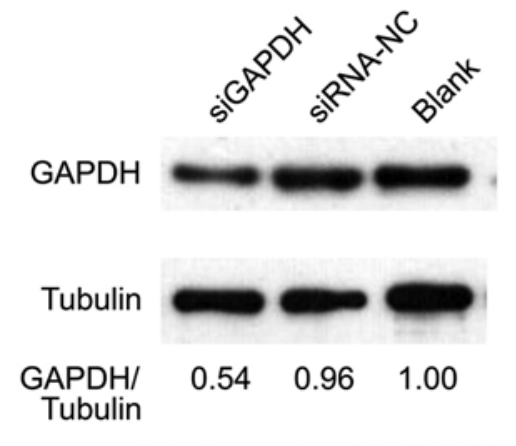

Lipofectamine 2000/siRNA

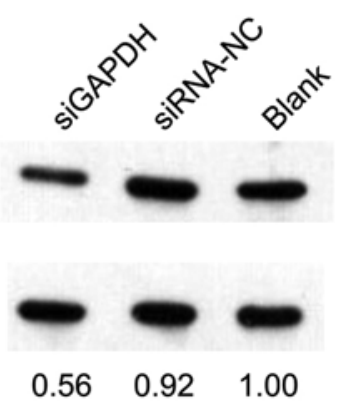

MnHAP/siRNA

Figure 6. Western blot analysis showing the expression of GAPDH protein at $48 \mathrm{~h}$ after a $4 \mathrm{~h}$ exposure to MnHAP/siRNA-GAPDH, MnHAP/siRNANC, untransfected cells (Blank) or Lipofectamine 2000/siRNA-GAPDH, Lipofectamine 2000/siRNA-NC, untransfected cells (Blank), respectively. Total tubulin levels were used as loading controls.

revealed that the proportion of FAM-positive cells increased remarkably following transfection with MnHAP/siRNA and Lipofectamine 2000/siRNA complexes compared to that with siRNA alone. According to the quantitative analysis shown in Fig. 3, the transfection efficiency of the group treated with FAM-labeled siRNA was $<0.50 \%(0.48 \pm 0.02 \%)$, whereas the efficiency was increased significantly for the MnHAP/siRNA treatment group $(96.49 \pm 0.34 \%)$, which is almost the same as that of Lipofectamine 2000/siRNA $(94.42 \pm 0.50 \%)$. The

FAM-positive cells in each group were observed and counted using fluorescence microscopy (Fig. 4A-C). The morphological features of the positive cells were confirmed and the positive cell percentages were in agreement with those from flow cytometry analyses.

Cytotoxicity of MnHAP/siRNA complexes. MTT assays were performed on BEL-7402 cells to evaluate the cytotoxicity of MnHAP. The results showed that the cell viability following exposure to MnHAP/siRNA complex was $81.60 \pm 2.00 \%$, whereas for the commercially available transfection agent Lipofectamine 2000/siRNA complex, the cell viability dropped to $64.40 \pm 1.69 \%$ (Fig. 5). As siRNA itself had no obvious cytotoxic effect on BEL-7402 cells, the cytotoxicity of MnHAP/ siRNA and Lipofectamine 2000/siRNA complexes were quite possibly due to MnHAP and Lipofectamine 2000 themselves. Even so, MnHAP was less toxic than Lipofectamine 2000 as demonstrated in Fig. 5.

The cytoxicity of MnHAP is similar to that of nHAP according to a previous study of BEL-7402 cells in which the cytotoxic effect was attributed to inhibition of proliferation and induction of apoptosis by nHAP (29). Therefore, our modification by PEI did not change the cytotoxicity of nHAP. However, in some studies, HAP without nano-processing was viewed as a non-toxic biocompatible biomaterial $(5,30)$. It has been reported that HAP cytotoxicity seemed to be related to its various physical characteristics including size, shape, charge and surface area as well as to the cell lines used, and that the degree of cell toxicity is positively associated with the amount of HAP taken up into cells (31). Compared to micro-size HAP, nano-sized nHAP particles are much more easily taken up by cells. The intracellular nHAP results in the rapid release of calcium, and this is the main reason for $\mathrm{nHAP}$ cytotoxicity (32).

Detection of GAPDH protein expression by western blot. In this study, we found that MnHAP could bind siRNA to form nano-complexes capable of knocking down GAPDH expression in BEL-7402 cells in vitro. Fig. 6, shows western blot analysis using tubulin as an internal control; GAPDH protein levels were lower when MnHAP delivered siRNA-GAPDH compared to the control siRNA-NC. Lipofectamine 2000 was similarly effective. The strong gene silencing activity of MnHAP loaded with siRNA-GAPDH is probably due to the high stability of complexes in the culture medium. 
In conclusion, we have successfully developed MnHAP, a novel transfection vector that can carry siRNA into BEL-7402 cells efficiently. This study shows that an MnHAP emulsion is stable for a longer time compared with that of nHAP, and this ensures its successful binding to siRNA for transfection. The high transfection efficiency and decreased cytotoxicity relative to Lipofectamine 2000 support that MnHAP may be a good candidate vector for biological and medical research. To further explore its biological properties and the potential medical applications, we will conduct further studies on the modification of MnHAP with ligands and on the delivery of other functional genes to target specific organs.

\section{Acknowledgements}

We thank Spandidos Language Editing Service of Spandidos Publications for their good comments, which were valuable for revising and improving our manuscript. This study was supported by grants from the Doctoral Program of Higher Education of China (grant no. 20120171110080), Science and Technology Planning Project of Guangdong Province, China (social development no. 2010B031100009), the general Program of National Natural Science Foundation of China (grant no. 30872996), Medical Scientific Research Foundation of Guangdong Province, China (grant no: A2012187).

\section{References}

1. Elbashir SM, Harborth J, Lendeckel W, Yalcin A, Weber K and Tuschl T: Duplexes of 21-nucleotide RNAs mediate RNA interference in cultured mammalian cells. Nature 411: 494-498, 2001.

2. Akhtar S and Benter IF: Nonviral delivery of synthetic siRNAs in vivo. J Clin Invest 117: 3623-3632, 2007.

3. Yi Y, Hahm SH and Lee KH: Retroviral gene therapy: Safety issues and possible solutions. Curr Gene Ther 5: 25-35, 2005.

4. Douglas KL: Toward development of artificial viruses for gene therapy: A comparative evaluation of viral and non-viral transfection. Biotechnol Prog 24: 871-883, 2008.

5. Berlin A, Cohen JL and Goldberg DJ: Calcium hydroxylapatite for facial rejuvenation. Semin Cutan Med Surg 25: 132-137, 2006

6. Afifi AM, Gordon CR, Pryor LS, Sweeney W, Papay FA and Zins JE: Calcium phosphate cements in skull reconstruction: A meta-analysis. Plast Reconstr Surg 126: 1300-1309, 2010.

7. Aoki H, Aoki H, Kutsuno T, Li W and Niwa M: An in vivo study on the reaction of hydroxyapatite-sol injected into blood. J Mater Sci Mater Med 11: 67-72, 2000.

8. Ashokan A, Chandran P, Sadanandan AR, Koduri CK, Retnakumari AP, Menon D, Nair S and Koyakutty M: Development and haematotoxicological evaluation of doped hydroxyapatite based multimodal nanocontrast agent for near-infrared, magnetic resonance and X-ray contrast imaging. Nanotoxicology 6: 652-666, 2012

9. Hou CH, Hou SM, Hsueh YS, Lin J, Wu HC and Lin FH: The in vivo performance of biomagnetic hydroxyapatite nanoparticles in cancer hyperthermia therapy. Biomaterials 30: 3956-3960, 2009.

10. Li G, Ye L, Pan J, et al: Antitumoural hydroxyapatite nanoparticles-mediated hepatoma-targeted trans-arterial embolization gene therapy: In vitro and in vivo studies. Liver Int 32: 998-1007, 2012 .

11. Matsumoto T, Okazaki M, Inoue M, Yamaguchi S, Kusunose T, Toyonaga T, Hamada Y and Takahashi J: Hydroxyapatite particles as a controlled release carrier of protein. Biomaterials 25: 3807-3812, 2004.
12. Roy ME and Nishimoto SK: Matrix Gla protein binding to hydroxyapatite is dependent on the ionic environment: Calcium enhances binding affinity but phosphate and magnesium decrease affinity. Bone 31: 296-302, 2002

13. Uskoković V and Uskoković DP: Nanosized hydroxyapatite and other calcium phosphates: Chemistry of formation and application as drug and gene delivery agents. J Biomed Mater Res B Appl Biomater 96: 152-191, 2011.

14. Gorbunoff MJ and Timasheff SN: The interaction of proteins with hydroxyapatite. III. Mechanism. Anal Biochem 136: 440-445, 1984.

15. Brigger I, Dubernet C and Couvreur P: Nanoparticles in cancer therapy and diagnosis. Adv Drug Deliv Rev 54: 631-651, 2002.

16. Khosravi-Darani K, Mozafari MR, Rashidi L and Mohammadi M: Calcium based non-viral gene delivery: An overview of methodology and applications. Acta Med Iran 48: 133-141, 2010.

17. Zhao Y, Zhang Y, Ning F, Guo D and Xu Z: Synthesis and cellular biocompatibility of two kinds of HAP with different nanocrystal morphology. J Biomed Mater Res B Appl Biomater 83: 121-126, 2007.

18. Puvvada N, Panigrahi PK and Pathak A: Room temperature synthesis of highly hemocompatible hydroxyapatite, study of their physical properties and spectroscopic correlation of particle size. Nanoscale 2: 2631-2638, 2010.

19. Sokolova VV, Radtke I, Heumann R and Epple M: Effective transfection of cells with multi-shell calcium phosphate-DNA nanoparticles. Biomaterials 27: 3147-3153, 2006.

20. Chen L, Mccrate JM, Lee JC and Li H: The role of surface charge on the uptake and biocompatibility of hydroxyapatite nanoparticles with osteoblast cells. Nanotechnology 22: 105708, 2011.

21. Slósarczyk A, Szymura-Oleksiak J and Mycek B: The kinetics of pentoxifylline release from drug-loaded hydroxyapatite implants. Biomaterials 21: 1215-1221, 2000.

22. Liu T, Tang A, Zhang G, Chen Y, Zhang J, Peng S and Cai Z: Calcium phosphate nanoparticles as a novel nonviral vector for efficient transfection of DNA in cancer gene therapy. Cancer Biother Radiopharm 20: 141-149, 2005.

23. Poulain L, Ziller C, Muller CD, Erbacher P, Bettinger T, Rodier JF and Behr JP: Ovarian carcinoma cells are effectively transfected by polyethylenimine (PEI) derivatives. Cancer Gene Ther 7: 644-652, 2000.

24. Liu C, Zhang P, Zhai X, Tian F, Li W, Yang J, Liu Y, Wang H, Wang $\mathrm{W}$ and Liu W: Nano-carrier for gene delivery and bioimaging based on carbon dots with PEI-passivation enhanced fluorescence. Biomaterials 33: 3604-3613, 2012.

25. Lungwitz U, Breunig $M$, Blunk $T$ and Göpferich $A$ : Polyethylenimine-based non-viral gene delivery systems. Eur J Pharm Biopharm 60: 247-266, 2005.

26. Godbey WT, Wu KK and Mikos AG: Size matters: Molecular weight affects the efficiency of poly(ethylenimine) as a gene delivery vehicle. J Biomed Mater Res 45: 268-275, 1999.

27. von Harpe A, Petersen H, Li Y and Kissel T: Characterization of commercially available and synthesized polyethylenimines for gene delivery. J Control Release 69: 309-322, 2000.

28. Ashokan A, Menon D, Nair S and Koyakutty M: A molecular receptor targeted, hydroxyapatite nanocrystal based multi-modal contrast agent. Biomaterials 31: 2606-2616, 2010.

29. Liu ZS, Tang SL and Ai ZL: Effects of hydroxyapatite nanoparticles on proliferation and apoptosis of human hepatoma BEL-7402 cells. World J Gastroenterol 9: 1968-1971, 2003.

30. Kveton JF and Coelho DH: Hydroxyapatite cement in temporal bone surgery: a 10 year experience. Laryngoscope 114: 33-37, 2004.

31. Gratton SE, Ropp PA, Pohlhaus PD, Luft JC, Madden VJ, Napier ME and DeSimone JM: The effect of particle design on cellular internalization pathways. Proc Natl Acad Sci USA 105: 11613-11618, 2008

32. Motskin M, Wright DM, Muller K, Kyle N, Gard TG, Porter AE and Skepper JN: Hydroxyapatite nano and microparticles: Correlation of particle properties with cytotoxicity and biostability. Biomaterials 30: 3307-3317, 2009. 